for calculation in this case to be a ring of $21 \mathrm{~mm}$. in diameter), provides the inductance. The anode current is fed to this ring at a voltage node. The arrangement can be seen from Fig. 1 .

It was hoped with this valve to obtain diode oscillations ${ }^{3}$ of the order of $30 \mathrm{~cm}$. without a magnetic field, but having failed to detect any, owing perhaps to the lack of exact symmetry in the apparatus, a magnetic field was applied parallel to the electrodes. Weak oscillations could be detected with a Lecher wire system coupled capacitatively to the valve, together with rectifier and sensitive galvanometer.

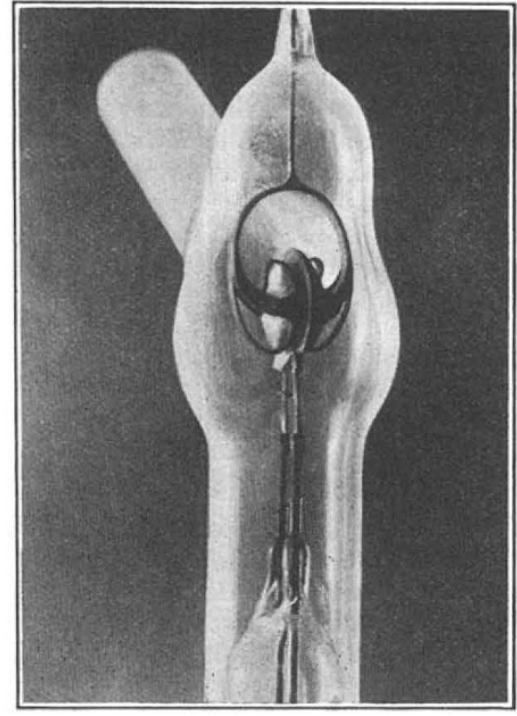

Frg. 1. Parallel-plane diode magnetron.

Until a further valve is built, little more can be said than :

(1) the oscillations occur where the magnetic field starts to cause a reduction in the anode current,

(2) the oscillation intensity falls off as the direction of the magnetic field is altered from that parallel to the electrodes, to that perpendicular to the plane of the electrodes.

(3) the wave-length of the oscillations is of the order of that determined by the oscillating circuit (the anode plates plus connecting wire).

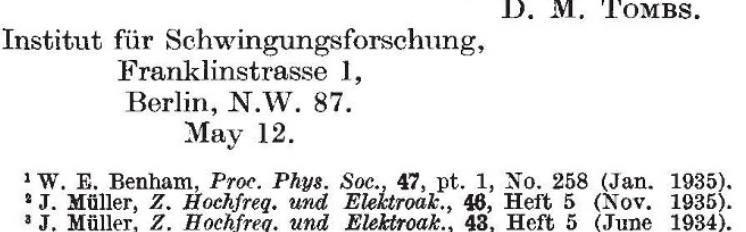

\section{Collisional Friction Frequency in the Ionosphere at Allahabad}

APPLETON $^{1}$ has shown that for the ordinary ray the integrated absorption coefficient $k$ for absorption in the deviating region (that is, in the region where $\mu$ approaches zero) may be calculated from the following formula :

$$
-2 \int_{\mu=1}^{\mu=0} \underset{\mu d h}{k=1}=\ln p_{0}=-\frac{\nu}{2 c}\left(P_{0}^{\prime}-P_{0}\right), . .
$$

where $\nu$, the collisional friction frequency per electron per second, is assumed to be constant throughout the deviating region; $p_{0}$ is the reflection coefficient for the ordinary ray; $P_{0}^{\prime}$ is the group path; and $P_{0}$ is the optical path of the wave.

Late in the night after the magneto-ionic splitting has taken place and the ordinary ray has undergone much group retardation, we are justified in assuming that

$$
\Delta\left(\ln p_{0}\right)=-\frac{\nu}{2 c} \Delta\left(P_{0}^{\prime}\right) \cdot \cdot \cdot
$$

Thus if we plot $\ln p_{0}$ against the equivalent height $P_{0}^{\prime} / 2$, we should expect a straight line, the slope of which will give us the value of $\nu$. The observations made in this laboratory during last winter show the validity of formula (2) and give an average value of $\nu$ of $1 \cdot 2 \times 10^{4}$ per electron per second.

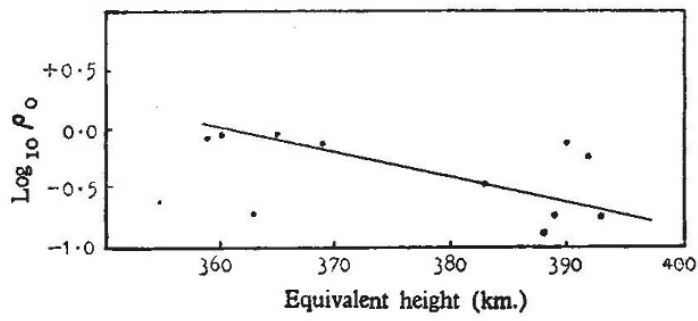

FiG. 1. F-layer reflections, March 1, 1936, time 03190402 I.S.T.

In Fig. $1, \log _{10} \rho_{0}$ has been plotted against the equivalent height for reflections from the $F^{\prime}$-layer. $v$ comes out to be $1.2 \times 10^{4}$ per electron per second.

The value of $v$ has also been determined by similar methods in England by Eckersley ${ }^{2}$ and Farmer and Ratcliffe ${ }^{3}$. Eckersley has found $v=3.6 \times 10^{3}$ in the daytime for the $F$-region, while Farmer and Ratcliffe's night-time value of $v$ for the $F$-region is $2 \cdot 1 \times 10^{3}$ and the daytime value is $1.2 \times 10^{3}$ per electron per second. Thus we see that the value found by us differs considerably from that found by workers in England. At present it is not possible to account for this difference, unless more data extending over a large period are available.

Further observations are being made and details will be published elsewhere.

G. R. Toshniwal.

B. D. Pant,

R. R. BAJPAI.

Physical Laboratory,

University, Allahabad. May 7.

1 Appleton, NATURE, 122, 879 (1928); 135, 618 (1935).

(1935).

${ }^{3}$ Farmer and Ratcliffe, Proc. Roy. Soc., A, 151, 370 (1935).

\section{Sensitivity of Photographic Plates in the Region $\lambda \lambda$ 2500-2100 A.}

IT is well known that the sensitivity of an ordinary photographic emulsion begins to fall rapidly just below $\lambda 2500 \mathrm{~A}$., and is very Iow at $\lambda 2100 \mathrm{~A}$. This fall is due to the absorption of short-wave radiation by the gelatin of the emulsion. It is commonly stated ${ }^{x}$ that higher sensitivity in this region may be obtained by greatly reducing the gelatin content of the emulsion, as in the Schumann plate, or by bathing an ordinary plate in a fluorescent substance which can 